\title{
Optimal control of Zika virus infection by vector elimination, vector-to-human and human-to-human contact reduction
}

\author{
Nonthamon Chaikham ${ }^{1,2}$ and Wannika Sawangtong ${ }^{1,2^{*}}$ (D)
}

\author{
"Correspondence: \\ wannika.saw@mahidol.ac.th \\ 'Department of Mathematics, \\ Faculty of Science, Mahidol \\ University, 272 Rama VI Road, \\ Bangkok, 10400, Thailand \\ ${ }^{2}$ Centre of Excellence in \\ Mathematics, Commission on \\ Higher Education, 328 Sri Ayuthya \\ Road, Bangkok, 10400, Thailand
}

\begin{abstract}
The recent outbreak of mosquito-borne Zika virus (ZIKV) in Brazil, with estimated cases surpassing 1.5 million, has gained attention due to its rapid spread and neurological complications associated with the infection such as microcephaly and Guillain-Barré syndrome. Beside vector transmission, primarily via Aedes mosquitoes, sexual transmission also contributes to the virus outbreak. The epidemiological patterns of these viruses suggest that Zika virus could cause other outbreaks, particularly in tropical regions with high vector concentration. To plan and prepare for a counter-control measure, it is important to study the previous cases. Hence, the control model is proposed from the deterministic model of Zika virus infection. Regarding control measures, the control functions for these strategies; vector elimination, vector-to-human contact reduction, and human-to-human contact reduction are introduced into the system. The necessary conditions for the optimal controls are determined using Pontryagin's maximum principle and the optimality system is solved using Rung-Kutta fourth order scheme. Consequently, numerical results of the system with control and the system without control are shown and discussed.
\end{abstract}

Keywords: Zika virus; epidemic model; Pontryagin's maximum principle; optimal control; mosquito; sexual transmission

\section{Introduction}

Zika virus (ZIKV) is a mosquito-borne virus first discovered in Africa, named after the Zika forest, located in Uganda. This virus infects human primarily via Aeges mosquitoes as the transmission vector. These mosquitoes are widely populated in tropical regions and also transmit other arboviruses such as dengue and chikungunya. The virus infection is typically accompanied by mild illness such as fever, skin rash, malaise, headache, muscle pain, and joint pain [1]. Despite being discovered since 1947, only 14 cases of human infection were reported before 2007. This arbovirus started gaining attention since the outbreak in Yap Island and French Polynesia, particularly the latter outbreak with more than 30,000 estimated cases attending the health care facilities. Moreover, there are reports on the connection between the increasing number of some neurological complications, microcephaly and Guillain-Barré Syndrome (GBS) for instance, and ZIKV infections in French Polynesia [2].

(c) The Author(s) 2017. This article is distributed under the terms of the Creative Commons Attribution 4.0 International License (http://creativecommons.org/licenses/by/4.0/), which permits unrestricted use, distribution, and reproduction in any medium, provided you give appropriate credit to the original author(s) and the source, provide a link to the Creative Commons license, and indicate if changes were made. 
The recent ZIKV outbreak in Brazil with over 1.5 million estimated cases from 2015 to 2016 received significant attention globally. The main reasons are its large number of infections, rapid transmission, and the increasing in the number of reported microcephaly coincided with the infection. The incidence became public health emergency and followed by the warning announcement from the World Health Organization(WHO).

Apart from the vector-mediated transmission, a human can also be infected by the Zika virus through sexual transmission. The virus has been detected in blood and semen [3]. There is a report of virus persisting in urine and semen after it disappeared from serum [4]. There are reported cases of ZIKV transmitted through sexual intercourse, and the virus transmitted from a man even before the onset of symptoms in one case [5]. An article studied on the Zika incidence of both genders in Rio [6] also suggests that sexual transmission could affect the overall outbreak. Consequently, mainly focusing on reducing mosquito population or the contacts between human and mosquitoes might not be sufficient. Hence, the measure against sexual transmission of the virus should be considered.

There is a possibility that ZIKV outbreak may emerge again in the future, especially in high vector concentration areas. To understand the dynamics and predict the situation in advance, mathematical models of Zika virus infection [2] that have previously been published should be studied. Beside vector transmission, the model proposed by Gao et al. [7] considers the direct transmission from exposed, symptomatically infected, and convalescent human to susceptible human via sexual contacts. Optimal control is also an important mathematical method deciding a strategy regarding epidemic control with provided scenarios [8-10]. There are some studies of the optimal control of several diseases such as dengue [11], chikungunya [12], and HIV [13]. The results show that optimal control helps in reducing the number of infected individuals and the spread of the virus. Hence, the optimal control problem will be derived, with an attempt to minimize the number of infected individuals as well as the cost of implementing the control strategy. There were suggestions of mosquito elimination, such as using insecticides, and human-to-mosquito contact reduction, such as using insect repellents or mosquito nets, as the control measures for Dengue epidemic $[11,14,15]$. Lastly, there is a suggestion of abstaining from sexual activity or consistently using protection during sexual intercourse [16]. This could be referred as human-to-human contact reduction.

Hence, the main goal of this work is to study a Zika virus infection model and propose an optimal control problem as previously stated. The control parameters introduced here are vector elimination, vector-to-human contact reduction, and human-to-human contact reduction. The mathematical model, consisting of vector transmission and sexual transmission, is from the study proposed by Gao et al. [7]. The control model is constructed by including control parameters into the model system, then analyzing the model using optimal control theory. The necessary conditions that an optimal control and corresponding state must satisfy will be determined using Pontryagin's maximum principle. After that, the optimality system of the optimal control problem will be shown. Lastly, the numerical results shall be shown and discussed sequentially.

\section{Mathematical model}

The Zika virus infection model with mosquito-borne and sexual transmission is proposed by Gao et al. [7]. In the human-mosquito model, the total human or host population at time $t$ or $N_{h}(t)$, is partitioned into six classes: susceptible $S_{h}(t)$, exposed $E_{h}(t)$, 
symptomatically infected $I_{h 1}(t)$, convalescent $I_{h 2}(t)$, asymptomatically infected $A_{h}(t)$, and recovered $R_{h}(t)$. The total mosquito or vector population $N_{v}(t)$ is partitioned into three classes: susceptible $S_{v}(t)$, exposed $E_{v}(t)$, and infectious $I_{v}(t)$. Thus, the total host population $N_{h}=S_{h}+E_{h}+I_{h 1}+I_{h 2}+A_{h}+R_{h}$ and the total vector population $N_{v}=S_{v}+E_{v}+I_{v}$, where both are assumed to be constant. Therefore, the Zika virus infection model is governed by a system of differential equations as follows:

$$
\begin{aligned}
\frac{d S_{h}}{d t} & =-a b \frac{I_{v}}{N_{h}} S_{h}-\beta \frac{K E_{h}+I_{h 1}+\tau I_{h 2}}{N_{h}} S_{h}, \\
\frac{d E_{h}}{d t} & =\theta\left(a b \frac{I_{v}}{N_{h}} S_{h}+\beta \frac{K E_{h}+I_{h 1}+\tau I_{h 2}}{N_{h}} S_{h}\right)-\alpha_{h} E_{h}, \\
\frac{d I_{h 1}}{d t} & =\alpha_{h} E_{h}-\gamma_{h 1} I_{h 1}, \\
\frac{d I_{h 2}}{d t} & =\gamma_{h 1} I_{h 1}-\gamma_{h 2} I_{h 2}, \\
\frac{d A_{h}}{d t} & =(1-\theta)\left(a b \frac{I_{v}}{N_{h}} S_{h}+\beta \frac{K E_{h}+I_{h 1}+\tau I_{h 2}}{N_{h}} S_{h}\right)-\gamma_{h} A_{h}, \\
\frac{d R_{h}}{d t} & =\gamma_{h 2} I_{h 2}+\gamma_{h} A_{h}, \\
\frac{d S_{v}}{d t} & =\mu_{v} N_{v}-a c \frac{\eta E_{h}+I_{h 1}}{N_{h}} S_{v}-\mu_{v} S_{v}, \\
\frac{d E_{v}}{d t} & =a c \frac{\eta E_{h}+I_{h 1}}{N_{h}} S_{v}-\left(\alpha_{v}+\mu_{v}\right) E_{v}, \\
\frac{d I_{v}}{d t} & =\alpha_{v} E_{v}-\mu_{v} I_{v} .
\end{aligned}
$$

In the system, a susceptible human can be infected either through the bite of an infectious mosquito or sexual contacts with an exposed, symptomatically infected, and convalescent human. The exposed human becomes symptomatically infected after the virus incubation period. The exposed and symptomatically infected human can sexually transmit the disease to both susceptible human and mosquitoes, whereas the symptomatically infected class is more contagious due to the higher load of viremia and virusemenia [17]. After the end of viremia period, the symptomatically infected individual enters convalescent phase. In this phase, the virus still appears in semen but disappears from the serum. Hence a convalescent individual can pass the virus to a susceptible human but cannot transmit to mosquitoes. The end of the viremic period is assumed to coincide with the disappearance of symptoms in symptomatically infected individuals. The asymptomatically infected human is assumed to be noncontagious to both human and vector. Furthermore, the death and birth rate of human are ignored since the epidemiological period is much shorter than a human lifespan. All parameter descriptions are summarized in Table 1.

\section{Optimal control problem}

The control strategy for the outbreak is proposed considering campaigns and suggestions from $\mathrm{WHO}$ and health organizations regarding Zika virus infection. Considering the vector transmission and sexual transmission, three control parameters are introduced for constructing the control model. The control variable $u_{1}(t)$ is the use of preventive measures such as insect repellent or mosquito net to reduce the contacts between human and 
Table 1 Model parameters

\begin{tabular}{llcc}
\hline Parameters & Description & Value & Reference \\
\hline$a$ & Biting rate of mosquito (number of bites per mosquito per day) & 0.5 & {$[18]$} \\
$b$ & $\begin{array}{l}\text { Probability of transmission from an infectious mosquito to a susceptible } \\
\text { human per bite (dimensionless) }\end{array}$ & 0.4 & {$[18]$} \\
$c$ & $\begin{array}{l}\text { Probability of transmission from a symptomatically infected human to a } \\
\text { susceptible mosquito per bite (dimensionless) }\end{array}$ & 0.5 & {$[19]$} \\
$\beta$ & $\begin{array}{l}\text { Transmission rate from a symptomatically infected humans to } \\
\text { susceptible humans (per day) }\end{array}$ & 0.05 & {$[7]$} \\
$\kappa$ & Relative human-to-human transmissibility of exposed to & 0.6 & {$[7]$} \\
& symptomatically infected humans (dimensionless) & & \\
$\tau$ & Relative human-to-human transmissibility of convalescent to & 0.3 & {$[7]$} \\
$\eta$ & symptomatically infected humans (dimensionless) & 0.1 & {$[7]$} \\
$\eta$ & Relative human-to-mosquito transmission probability of exposed & & \\
$\theta(\%)$ & humans to symptomatically infected human (dimensionless) & 18 & {$[20]$} \\
$1 / \alpha_{h}$ & Proportion of symptomatic infections (dimensionless) & 5 & {$[21]$} \\
$1 / \alpha_{V}$ & Intrinsic incubation period (days) & 10 & {$[18]$} \\
$1 / \gamma_{h 1}$ & Extrinsic incubation period (days) & 5 & {$[21]$} \\
$1 / \gamma_{h 2}$ & Period of acute phase (days) & 20 & {$[4]$} \\
$1 / \gamma_{h}$ & Period of convalescent phase (days) & 7 & {$[7]$} \\
$1 / \mu_{V}$ & Period of asymptomatic infection (days) & 14 & {$[18,19]$} \\
\hline
\end{tabular}

mosquito. The control variable $u_{2}(t)$ is the measure of abstaining from sexual activity or consistent use of protections in sexual activity. The control variable $u_{3}(t)$ is the use of pesticides or chemicals at mosquito breeding sites to eliminate or reduce the total number of mosquitoes. Consequently, from control strategy, the forces of infection in the human population are reduced by the factors of $\left(1-u_{1}(t)\right)$ and $\left(1-u_{2}(t)\right)$. Similarly, the force of infection in the mosquito population is reduced by a factor of $\left(1-u_{1}(t)\right)$. Moreover, the mosquito birth rate is reduced by a factor of $\left(1-u_{3}(t)\right)$. It is also assumed that the mosquito death rate increases by $r_{0} u_{3}(t)$, where $r_{0}>0$. Therefore, the dynamics of the system (1) with control components is governed by the system of differential equations as follows:

$$
\begin{aligned}
\frac{d S_{h}}{d t} & =-a b \frac{I_{v}}{N_{h}} S_{h}\left(1-u_{1}\right)-\beta \frac{K E_{h}+I_{h 1}+\tau I_{h 2}}{N_{h}} S_{h}\left(1-u_{2}\right), \\
\frac{d E_{h}}{d t} & =\theta\left(a b \frac{I_{v}}{N_{h}} S_{h}\left(1-u_{1}\right)+\beta \frac{K E_{h}+I_{h 1}+\tau I_{h 2}}{N_{h}} S_{h}\left(1-u_{2}\right)\right)-\alpha_{h} E_{h}, \\
\frac{d I_{h 1}}{d t} & =\alpha_{h} E_{h}-\gamma_{h 1} I_{h 1} \\
\frac{d I_{h 2}}{d t} & =\gamma_{h 1} I_{h 1}-\gamma_{h 2} I_{h 2} \\
\frac{d A_{h}}{d t} & =(1-\theta)\left(a b \frac{I_{v}}{N_{h}} S_{h}\left(1-u_{1}\right)+\beta \frac{K E_{h}+I_{h 1}+\tau I_{h 2}}{N_{h}} S_{h}\left(1-u_{2}\right)\right)-\gamma_{h} A_{h}, \\
\frac{d R_{h}}{d t} & =\gamma_{h 2} I_{h 2}+\gamma_{h} A_{h}, \\
\frac{d S_{v}}{d t} & =\mu_{v} N_{v}\left(1-u_{3}\right)-a c \frac{\eta E_{h}+I_{h 1}}{N_{h}} S_{v}\left(1-u_{1}\right)-\left(\mu_{v}+r_{0} u_{3}\right) S_{v}, \\
\frac{d E_{v}}{d t} & =a c \frac{\eta E_{h}+I_{h 1}}{N_{h}} S_{v}\left(1-u_{1}\right)-\left(\alpha_{v}+\mu_{v}+r_{0} u_{3}\right) E_{v}, \\
\frac{d I_{v}}{d t} & =\alpha_{v} E_{v}-\left(\mu_{v}+r_{0} u_{3}\right) I_{v} .
\end{aligned}
$$


Next, we define our objective function:

$$
J\left(u_{1}, u_{2}, u_{3}\right)=\int_{0}^{T}\left(A_{1} E_{h}+A_{2} I_{h}+A_{3} N_{v}+\frac{1}{2}\left(B_{1} u_{1}^{2}+B_{2} u_{2}^{2}+B_{3} u_{3}^{2}\right)\right) d t
$$

subject to the state system of (2).

Next, we will find the optimal controls, namely the controls that optimize our objective function. Regarding this work, we will find a set of controls that minimizes the number of exposed human, symptomatically infected human, and the total number of mosquitoes as well as the costs associated with implementing the control strategy. The constants $A_{1}$, $A_{2}$, and $A_{3}$, are the weighted constants associated with exposed human, symptomatically infected human, and the total number of mosquito, respectively. The constants $B_{1}, B_{2}$, and $B_{3}$ are the weighted constants of the control variables $u_{1}, u_{2}$ and $u_{3}$, respectively. The terms $\frac{1}{2} B_{1} u_{1}^{2}, \frac{1}{2} B_{2} u_{2}^{2}$, and $\frac{1}{2} B_{3} u_{3}^{2}$ are the costs associated with implementing each of the three controls. The cost related to the first control might be the expenses of using insect repellent, mosquito net, and herbal spray for instance. The cost related to second control might be the expenses of providing protections or warning leaflets about the safe sexual activity. The cost related to the last control could come from the expenses of using mosquito pesticides and the process implementation.

Let $u_{1}^{*}, u_{2}^{*}$, and $u_{3}^{*}$ be the optimal controls, we will find a set of control functions such that

$$
J\left(u_{1}^{*}, u_{2}^{*}, u_{3}^{*}\right)=\min J\left(u_{1}, u_{2}, u_{3}\right), \quad\left(u_{1}, u_{2}, u_{3}\right) \in U
$$

subject to the state system (2), where the control set $U$ is

$$
U=\left\{\left(u_{1}, u_{2}, u_{3}\right) \mid u_{i}(t) \text { is Lebesgue measurable on }[0,1], 0 \leq u_{i}(t) \leq 1, i=1,2,3\right\} .
$$

\subsection{Characterization of the control problem}

We will use Pontryagin's maximum principle [22] to find the optimal controls that satisfy the necessary conditions [23]. Firstly, the Lagrangian for the optimal control problem is defined by

$$
L=A_{1} E_{h}+A_{2} I_{h}+A_{3} N_{v}+1 / 2\left(B_{1} u_{1}^{2} B_{2} u_{2}^{2} B_{3} u_{3}^{2}\right)
$$

and the Hamiltonian $H$

$$
\begin{aligned}
H= & L\left(E_{h}, I_{h}, N_{v}, u_{1}, u_{2}, u_{3}\right)+\lambda_{1} \frac{d S_{h}}{d t}+\lambda_{2} \frac{d E_{h}}{d t}+\lambda_{3} \frac{d I_{h 1}}{d t}+\lambda_{4} \frac{d I_{h 2}}{d t} \\
& +\lambda_{5} \frac{d A_{h}}{d t}+\lambda_{6} \frac{d S_{v}}{d t}+\lambda_{7} \frac{d E_{v}}{d t}+\lambda_{8} \frac{d I_{v}}{d t} \\
= & A_{1} E_{h}+A_{2} I_{h 1}+A_{3} N_{v}+\frac{1}{2}\left(B_{1} u_{1}^{2}+B_{2} u_{2}^{2}+B_{3} u_{3}^{2}\right) \\
& +\lambda_{1}\left\{-a b \frac{I_{v}}{N_{h}} S_{h}\left(1-u_{1}\right)-\beta \frac{K E_{h}+I_{h 1}+\tau I_{h 2}}{N_{h}} S_{h}\left(1-u_{2}\right)\right\} \\
& +\lambda_{2}\left\{\theta\left(a b \frac{I_{v}}{N_{h}} S_{h}\left(1-u_{1}\right)+\beta \frac{K E_{h}+I_{h 1}+\tau I_{h 2}}{N_{h}} S_{h}\left(1-u_{2}\right)\right)-\alpha_{h} E_{h}\right\}
\end{aligned}
$$




$$
\begin{aligned}
& +\lambda_{3}\left\{\alpha_{h} E_{h}-\gamma_{h 1} I_{h 1}\right\}+\lambda_{4}\left\{\gamma_{h 1} I_{h 1}-\gamma_{h 2} I_{h 2}\right\} \\
& +\lambda_{5}\left\{(1-\theta)\left(a b \frac{I_{v}}{N_{h}} S_{h}\left(1-u_{1}\right)+\beta \frac{K E_{h}+I_{h 1}+\tau I_{h 2}}{N_{h}} S_{h}\left(1-u_{2}\right)\right)-\gamma_{h} A_{h}\right\} \\
& +\lambda_{6}\left\{\mu_{v} N_{v}\left(1-u_{3}\right)-a c \frac{\eta E_{h}+I_{h 1}}{N_{h}} S_{v}\left(1-u_{1}\right)-\left(\mu_{v}+r_{0} u_{3}\right) S_{v}\right\} \\
& +\lambda_{7}\left\{a c \frac{\eta E_{h}+I_{h 1}}{N_{h}} S_{v}\left(1-u_{1}\right)-\left(\alpha_{v}+\mu_{v}+r_{0} u_{3}\right) E_{v}\right\} \\
& +\lambda_{8}\left\{\alpha_{v} E_{v}-\left(\mu_{v}+r_{0} u_{3}\right) I_{v}\right\}
\end{aligned}
$$

where $\lambda_{1}, \lambda_{2}, \lambda_{3}, \lambda_{4}, \lambda_{5}, \lambda_{6}, \lambda_{7}$, and $\lambda_{8}$ are the adjoint variables satisfying the following adjoint system:

$$
\begin{aligned}
\lambda_{1}^{\prime}=-\frac{\partial H}{\partial S_{h}}= & \frac{1}{N_{h}}\left(\lambda_{1}-\lambda_{2} \theta-\lambda_{5}(1-\theta)\right)\left(a b I_{v}\left(1-u_{1}\right)+\beta\left(K E_{h}+I_{h 1}+\tau I_{h 2}\right)\left(1-u_{2}\right)\right), \\
\lambda_{2}^{\prime}=-\frac{\partial H}{\partial E_{h}}= & -A_{1}+\frac{K \beta}{N_{h}} S_{h}\left(1-u_{2}\right)\left(\lambda_{1}-\lambda_{2} \theta-\lambda_{5}(1-\theta)\right) \\
& +\left(\lambda_{2}-\lambda_{3}\right) \alpha_{h}+\frac{a c}{N_{h}} S_{v} \eta\left(1-u_{1}\right)\left(\lambda_{6}-\lambda_{7}\right), \\
\lambda_{3}^{\prime}=-\frac{\partial H}{\partial I_{h 1}}= & -A_{2}+\frac{\beta}{N_{h}} S_{h}\left(1-u_{2}\right)\left(\lambda_{1}-\lambda_{2} \theta-\lambda_{5}(1-\theta)\right) \\
& +\left(\lambda_{3}-\lambda_{4}\right) \gamma_{h 1}+\frac{a c}{N_{h}} S_{v}\left(1-u_{1}\right)\left(\lambda_{6}-\lambda_{7}\right), \\
\lambda_{4}^{\prime}=-\frac{\partial H}{\partial I_{h 2}}= & \frac{\beta \tau}{N_{h}} S_{h}\left(1-u_{2}\right)\left(\lambda_{1}-\lambda_{2} \theta-\lambda_{5}(1-\theta)\right)+\lambda_{4} \gamma_{h 2}, \\
\lambda_{5}^{\prime}=-\frac{\partial H}{\partial A_{h}}= & \lambda_{5} \gamma_{h}, \\
\lambda_{6}^{\prime}=-\frac{\partial H}{\partial S_{v}}= & -A_{3}+\frac{a c}{N_{h}}\left(\eta E_{h}+I_{h}\right)\left(1-u_{1}\right)\left(\lambda_{6}-\lambda_{7}\right)+\left(\mu_{v}+r_{0}\right) u_{3} \lambda_{6}, \\
\lambda_{7}^{\prime}=-\frac{\partial H}{\partial E_{v}}= & -A_{3}-\lambda_{6} \mu_{v}\left(1-u_{3}\right)+\lambda_{7}\left(\alpha_{v}+\mu_{v}+r_{0} u_{3}\right)-\lambda_{8} \alpha_{v}, \\
\lambda_{8}^{\prime}=-\frac{\partial H}{\partial I_{v}}= & -A_{3}+\frac{a b}{N_{h}} S_{h}\left(1-u_{1}\right)\left(\lambda_{1}-\lambda_{2} \theta-\lambda_{5}(1-\theta)\right) \\
& \left.-u_{3}\right)-\lambda_{8}\left(\mu_{v}+r_{0} u_{3}\right) .
\end{aligned}
$$

The transversality conditions (or boundary conditions) are

$$
\lambda_{i}(T)=0, \quad i=1,2, \ldots, 8, \text { where } T \text { is the end of the time period. }
$$

By the optimality condition, we have

$$
\frac{\partial H}{\partial u_{i}}=0, \quad i=1,2,3 \text {, at } u_{i} \Rightarrow u_{i}^{*}
$$

Thus,

$$
\frac{\partial H}{\partial u_{1}}=B_{1} u_{1}+\frac{a}{N_{h}}\left(b S_{h} I_{v}\left(\lambda_{1}-\lambda_{2} \theta-\lambda_{5}(1-\theta)\right)+c S_{v}\left(\eta E_{h}+I_{h 1}\right)\left(\lambda_{6}-\lambda_{7}\right)\right)=0,
$$




$$
\begin{aligned}
& \frac{\partial H}{\partial u_{2}}=B_{2} u_{2}+\frac{\beta S_{h}}{N_{h}}\left(K E_{h}+I_{h 1}+\tau I_{h 2}\right)\left(\lambda_{1}-\lambda_{2} \theta-\lambda_{5}(1-\theta)\right)=0, \\
& \frac{\partial H}{\partial u_{3}}=B_{3} u_{3}-\left(\lambda_{6}\left(\mu_{v} N_{v}+r_{0} S_{v}\right)-r_{0} \lambda_{7} E_{v}-r_{0} \lambda_{8} I_{v}\right)=0 .
\end{aligned}
$$

We obtain

$$
\begin{aligned}
& u_{1}^{*}=\max \left\{\min \left\{\frac{a}{B_{1} N_{h}}\left(b S_{h} I_{v}\left(-\lambda_{1}+\lambda_{2} \theta+\lambda_{5}(1-\theta)\right)+c S_{v}\left(\eta E_{h}+I_{h 1}\right)\left(\lambda_{7}-\lambda_{6}\right)\right), 1\right\}, 0\right\}, \\
& u_{2}^{*}=\max \left\{\min \left\{\frac{\beta S_{h}}{B_{2} N_{h}}\left(K E_{h}+I_{h 1}+\tau I_{h 2}\right)\left(-\lambda_{1}+\lambda_{2} \theta+\lambda_{5}(1-\theta)\right), 1\right\}, 0\right\}, \\
& u_{3}^{*}=\max \left\{\min \left\{\frac{1}{B_{3}}\left(\lambda_{6}\left(\mu_{v} N_{v}+r_{0} S_{v}\right)+r_{0} \lambda_{7} E_{v}+r_{0} \lambda_{8} I_{v}\right), 1\right\}, 0\right\} .
\end{aligned}
$$

Therefore, we obtain the optimality system which helps describe the behavior of the system with the optimal controls. The optimality system consists of the state system (2), initial conditions of the state variables, the adjoint system (7), the transversality conditions (8), and the characterization of the optimal control.

\section{Numerical results and discussion}

The main purpose of this research is to study the effect of the control strategy on the dynamics of the ZIKV outbreak. First, the optimality system is numerically solved using the iterative method with the Runge-Kutta fourth order scheme. The state system (2) is solved by the forward Runge-Kutta method with an initial guess, and the adjoint system (7) is solved by the backward Runge-Kutta method with the transversality condition [23]. For all simulations, some of the parameter values are from publications which are defined in Table 1. Other parameters are arbitrarily chosen with $r_{0}=0.01$, and $T=90$ days. For illustration purpose, the initial values of the state variables for solving system (1) are given by the authors. For the human group, the initial number of susceptible, exposed, symptomatically infected, convalescent, asymptomatically infected, and recovered individuals $\left(S_{h}(0), E_{h}(0), I_{h 1}(0), I_{h 2}(0), A_{h}(0), R_{h}(0)\right)$ is set by $(100,000,540,135,100,100,12)$. For the mosquito group, the initial number of susceptible, exposed, and infected vectors $\left(S_{v}(0), E_{v}(0), I_{v}(0)\right)$ is set by $(10,000,1,000,100)$. The weight constants are given as $A_{1}=0.05, A_{2}=0.05, A_{3}=0.05, B_{1}=10, B_{2}=10$, and $B_{3}=30$. For $B_{1}, B_{2}$, and $B_{3}$, which is based on the assumption that vector elimination or mosquito elimination is more expensive in terms of implementation costs than the other two controls. There are costs of pesticides and costs of operating the control, which might be time-consuming and laborintensive. On the contrary, providing insect repellent or other types of protections against mosquito's biting is easier and probably cheaper. Similarly, for sexual transmission, providing protection such as condoms or promoting the priority of protected sexual activity requires less money and resources. $T$ is chosen to be 90 days or about three months which is the average period of one season.

Generally, in the absence of vaccination or treatment, it is recommended that one use pesticides or avoid being bitten by mosquitoes [24]. Hence, we first consider the setting where there are two controls $u_{1}$ and $u_{3}$. We also consider the scenario where there is no vector elimination, or control $u_{3}$ since it might be dangerous to use pesticides in some area such as hospitals or living habitats for a long time. This can be represented by the 


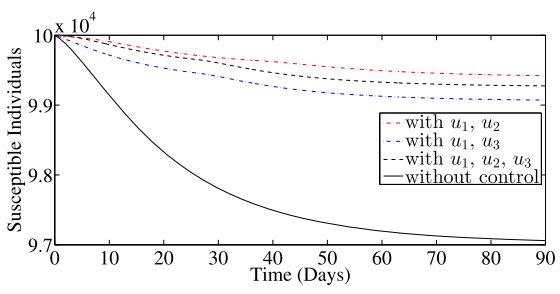

(a) Susceptible individuals

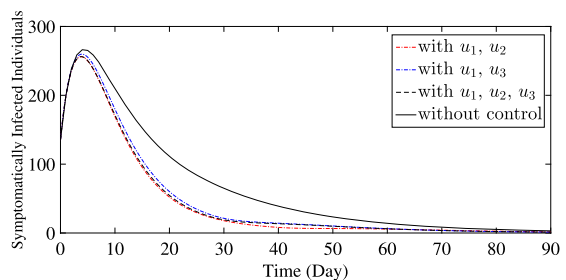

(c) Symptomatically infected individuals

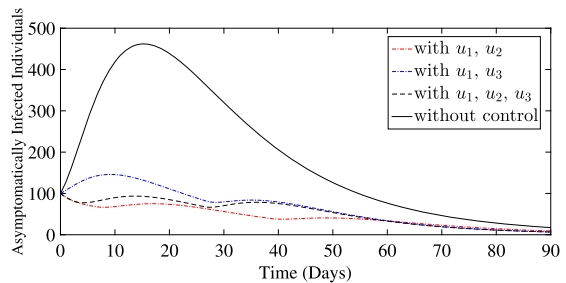

(e) Asymptomatically infected individuals

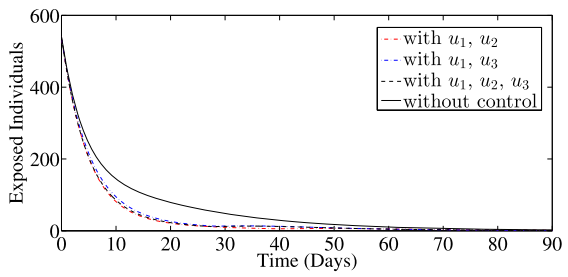

(b) Exposed individuals

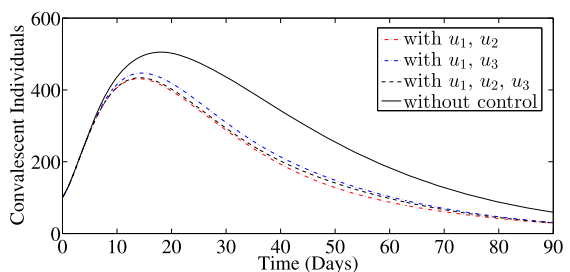

(d) Convalescent individuals

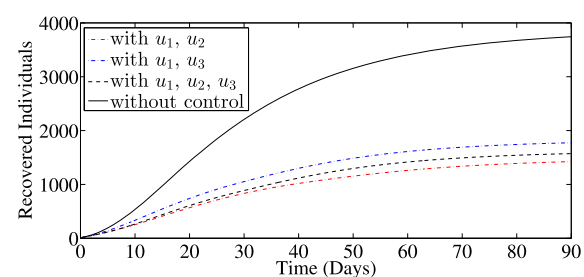

(f) Recovered Individuals

Figure 1 Number of individuals from each class. Number of individuals from the optimality system without control and the optimality systems with controls.

setting with only two controls, $u_{1}$ and $u_{2}$. Lastly, we consider the scenario where three controls are applied together. Therefore, the three scenarios are as follows: i) with controls $u_{1}$ and $u_{2}$, ii) with controls $u_{1}$ and $u_{3}$, and iii) with three controls $u_{1}, u_{2}$, and $u_{3}$. In practice, our strategy cannot be completely implemented, so the maximum value of each control is set to be 0.9 instead of 1 . Consequently, we simulated three different scenarios to the optimality system with controls and the optimality system without control. The numerical results for each class of individuals and vectors are illustrated. In every graph, each of the individuals without control is represented by a solid line. The individuals with controls are represented as follows: a red dash-dot line for the system with controls $u_{1}$ and $u_{2}$, a blue dash-dot line for the system with controls $u_{1}$ and $u_{3}$, and a black dashed line for the system with all three controls.

Figure 1 shows the number of susceptible individuals, exposed individuals, symptomatically infected individuals, convalescent individuals, asymptomatically infected individuals, and recovered individuals from the optimality system without control and the optimality system with controls. Figure 2 shows the number of susceptible vectors, exposed vectors, and infectious vectors from the optimality system without control and optimality system with control. Figure 3 shows the level of optimal controls $u_{1}, u_{2}$, and $u_{3}$ at a time from different scenarios.

From Figure 1, it could be seen that the system with $u_{1}$ and $u_{2}$, and the system with three controls give the best result. The number of exposed, symptomatically infected, and convalescent of both mentioned systems are lowest, which means the disease will die out 


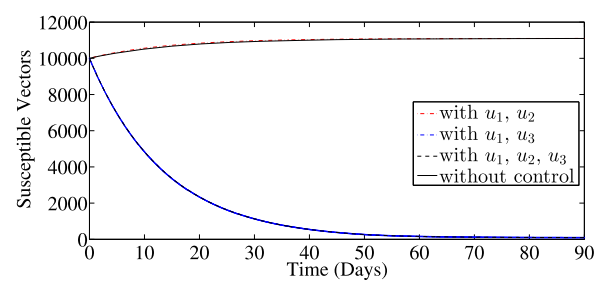

(a) Susceptible vectors

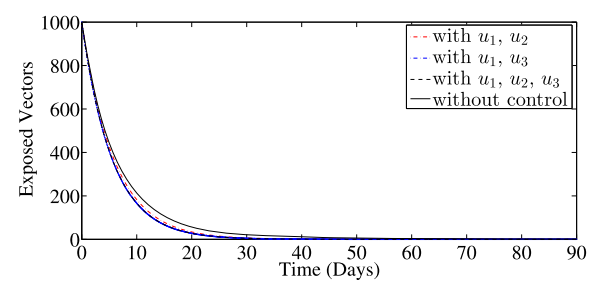

(b) Exposed vectors

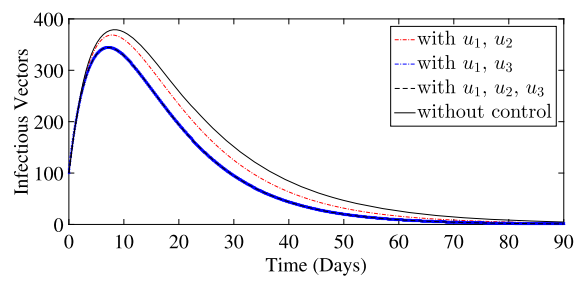

(c) Infectious vectors

Figure 2 Number of vectors from each class. Number of vectors from the optimality system without control and the optimality systems with controls.

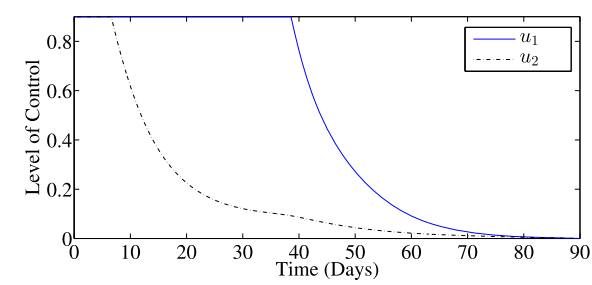

(a) $u_{1} \neq 0, u_{2} \neq 0, u_{3}=0$

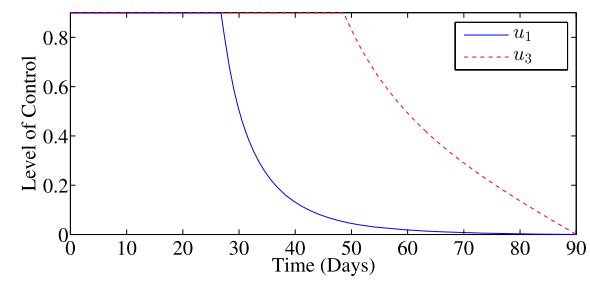

(b) $u_{1} \neq 0, u_{2}=0, u_{3} \neq 0$

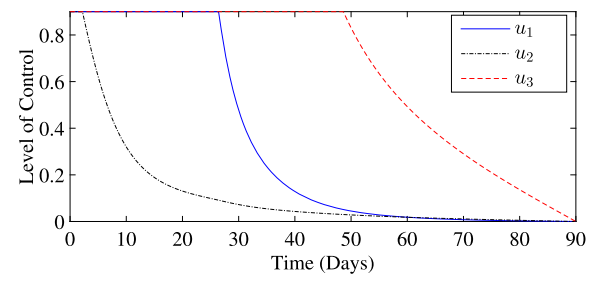

(c) $u_{1} \neq 0, u_{2} \neq 0, u_{3} \neq 0$

Figure 3 Level of the optimal controls. Level of the optimal controls of (a) scenario with two controls $\left(u_{1}, u_{2}\right),(\mathbf{b})$ scenario with two controls $\left(u_{1}, u_{3}\right)$, and $(\mathbf{c})$ scenario with three controls $\left(u_{1}, u_{2}, u_{3}\right)$.

faster than the other systems. From Figures $2 \mathrm{~b}-2 \mathrm{c}$, it can be seen that the system with $u_{1}$ and $u_{3}$, and the system with three controls give the best result with the lowest number of exposed and infectious vectors. This could mean that both systems are the most effective in reducing the number of mosquitoes.

From Figure 3, the control variable $u_{1}$ stays at the maximum level about 40 days for the system with $u_{1}, u_{2}$, and lasts 30 days for the system with $u_{1}, u_{3}$ and the system with three controls. Note that $E_{h}, I_{h 1}, E_{v}$, and $I_{v}$ also become very low by that time. For the second control, $u_{2}$ is at maximum level for several days, then decreases sharply within about the first week, which corresponds to the peak of the number of $I_{h 1}$. Lastly, the level of $u_{3}$ is at the maximum value until about day 50 before decreasing in every system setting. Note 


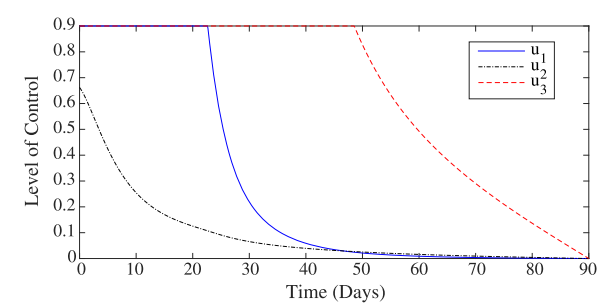

(a) $a_{1}=0.25$

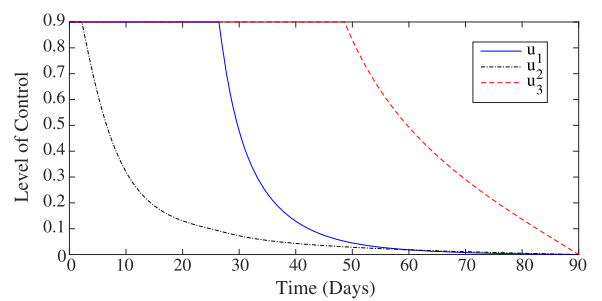

(b) $a_{2}=0.5$

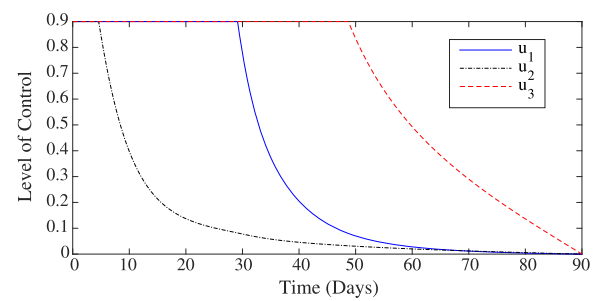

(c) $a_{3}=0.75$

Figure 4 Level of the optimal controls with different biting rates. Level of the optimal controls with the biting rate (a) $a=0.25$, (b) $a=0.5$, and (c) $a=0.75$.

that $u_{3}$ starts decreasing when the total population of mosquitoes almost vanishes since the control $u_{3}$ contributes the most to reducing the total population of mosquitoes.

Over the last few decades, Zika virus infection occurred in many countries, and the behavior of the disease transmission might be different in each area. Considering the mosquito biting rate as one of the factors of the disease, we investigate the effect of the mosquito's biting rate on the control variables. Therefore, the numerical results of the level of controls by different setting of biting rate are illustrated in Figure 4, where biting rate (a) is varied as $a_{1}=0.25, a_{2}=0.5$, and $a_{3}=0.75$. From Figures $4 \mathrm{a}-4 \mathrm{c}$, the level of $u_{1}$ stays at the maximum level about 23,26 , and 29 days for the setting $a_{1}, a_{2}$, and $a_{3}$, respectively. After that, the level of $u_{1}$ in each setting decreases sharply. The level of $u_{2}$ in the setting $a_{1}$ begins at 0.65 and gradually decreases as shown in the Figure $4 \mathrm{a}$. From Figures $4 \mathrm{~b}-4 \mathrm{c}$, the level of $u_{2}$ for the setting $a_{2}$ and $a_{3}$ stays at the maximum level for two and five days, respectively, and decreases after that. The results indicate that the increasing of the value of biting rate leads to an extension of the period at the beginning where the control $u_{1}$ and $u_{2}$ are at the maximum level. On the contrary, the level of $u_{3}$ is the same in each setting.

\section{Conclusions}

In this paper, we derived a control model from the deterministic model of the Zika virus infection which consists of mosquito-borne transmission and sexual transmission by Gao et al. [7]. The optimal control problem is proposed with three selected controls; vector elimination, vector-to-human contact reduction, human-to-human contact reduction. The optimal control problem is developed to minimize the number of exposed humans, symptomatically infected humans, the total number of vectors as well as the costs associated with implementing the controls. We use Pontryagin's maximum principle to determine the necessary conditions, find the optimality system of the model and hence find the solution to the optimal control problem. Numerical simulations comparing the systems without control and the system with controls are presented. It is shown that an optimal control strategy is more effective in reducing the infected humans and vectors than without con- 
trol strategy. From the numerical results, it is shown that time resource and human resource in implementing the strategy will be required mostly at the beginning of the period and then lessen over time. Additionally, the variation of the model parameter has an effect on the optimal controls. Our simulations imply that the areas with higher mosquito's biting rate require more efforts in controlling the disease. In conclusion, the behavior of the optimal controls should be changed according to the scenarios. This will help us to plan the strategy most efficiently. Furthermore, apart from mosquito elimination and protection against mosquito's biting which is the standard measures, the human-to-human contact reduction also contributes to the control of the outbreak. Besides using pesticides, using protection against mosquito contacts and sexual contacts could help in reducing the spread of Zika virus infection.

\footnotetext{
Acknowledgements

This research was supported by the Centre of Excellence in Mathematics, Commission on Higher Education, Thailand and Faculty of Science, Mahidol University, Thailand.
}

\section{Competing interests}

The authors declare that they have no competing interests.

\section{Authors' contributions}

WS provided the research idea, developed the control model, and verified the numerical results. NC solved the control model to simulate the results by MATLAB programming and wrote this article. All authors read and approved the final submitted version.

\section{Publisher's Note}

Springer Nature remains neutral with regard to jurisdictional claims in published maps and institutional affiliations.

Received: 31 January 2017 Accepted: 26 May 2017 Published online: 23 June 2017

\section{References}

1. Dasti, Jl: Zika virus infections: an overview of current scenario. Asian Pac. J. Trop. Med. 9(7), 621-625 (2016)

2. Kucharski, AJ, Funk, S, Eggo, RM, Mallet, H-P, Edmunds, WJ, Nilles, EJ: Transmission dynamics of Zika virus in island populations: a modelling analysis of the 2013-14 French Polynesia outbreak. PLoS Negl. Trop. Dis. 10(5), 0004726 (2016)

3. Atkinson, B, Hearn, P, Afrough, B, Lumley, S, Carter, D, Aarons, EJ, Simpson, AJ, Brooks, TJ, Hewson, R: Detection of Zika virus in semen. Emerg. Infect. Dis. 22(5), 940 (2016)

4. Musso, D, Roche, C, Robin, E, Nhan, T, Teissier, A, Cao-Lormeau, V-M: Potential sexual transmission of Zika virus. Emerg. Infect. Dis. 21(2), 359-361 (2015)

5. Foy, BD, Kobylinski, KC, Foy, JL, Blitvich, BJ, da Rosa, AT, Haddow, AD, Lanciotti, RS, Tesh, RB: Probable non-vector-borne transmission of Zika virus. Emerg. Infect. Dis. 17(5), 880-882 (2011)

6. Coelho, FC, Durovni, B, Saraceni, V, Lemos, C, Codeco, CT, Camargo, S, de Carvalho, LM, Bastos, L, Arduini, D, Villela, DA, Armstrong, M: Higher incidence of Zika in adult women than adult men in Rio de Janeiro suggests a significant contribution of sexual transmission from men to women. Int. J. Infect. Dis. 51, 128-132 (2016)

7. Gao, D, Lou, Y, He, D, Porco, TC, Kuang, Y, Chowell, G, Ruan, S: Prevention and control of Zika as a mosquito-borne and sexually transmitted disease: a mathematical modeling analysis. Sci. Rep. 6, 28070 (2016)

8. Gupta, N, Rink, R: Optimum control of epidemics. Math. Biosci. 18(3-4), 383-396 (1973)

9. Lashari, AA, Zaman, G: Optimal control of a vector borne disease with horizontal transmission. Nonlinear Anal., Real World Appl. 13(1), 203-212 (2012)

10. Yan, $X, Z$ Zou, Y, Li, J: Optimal quarantine and isolation strategies in epidemics control. World J. Model. Simul. 3(3), 202-211 (2007)

11. Rodrigues, HS, Monteiro, MTT, Torres, DF: Vaccination models and optimal control strategies to dengue. Math. Biosci. $247,1-12(2014)$

12. Moulay, D, Aziz-Alaoui, M, Kwon, H-D: Optimal control of chikungunya disease: larvae reduction, treatment and prevention. Math. Biosci. Eng. 9(2), 369-392 (2012)

13. Kirschner, D, Lenhart, S, Serbin, S: Optimal control of the chemotherapy of hiv. J. Math. Biol. 35(7), 775-792 (1997)

14. Thomé, RC, Yang, HM, Esteva, L: Optimal control of aedes aegypti mosquitoes by the sterile insect technique and insecticide. Math. Biosci. 223(1), $12-23$ (2010)

15. Aldila, D, Götz, T, Soewono, E: An optimal control problem arising from a dengue disease transmission model. Math. Biosci. 242(1), 9-16 (2013)

16. Update: interim guidelines for prevention of sexual transmission of Zika virus - United States, 2016. Centers for Disease Control and Prevention (CDC). https://emergency.cdc.gov/han/han00388.asp (2016)

17. Hills, SL: Transmission of Zika virus through sexual contact with travelers to areas of ongoing transmission continental United States, 2016. Morb. Mort. Wkly. Rep. 65, 215-216 (2016) 
18. Andraud, M, Hens, N, Marais, C, Beutels, P: Dynamic epidemiological models for dengue transmission: a systematic review of structural approaches. PLoS ONE 7(11), 49085 (2012)

19. Chikaki, E, Ishikawa, H: A dengue transmission model in Thailand considering sequential infections with all four serotypes. J. Infect. Dev. Ctries. 3(9), 711-722 (2009)

20. Duffy, MR, Chen, T-H, Hancock, WT, Powers, AM, Kool, JL, Lanciotti, RS, Pretrick, M, Marfel, M, Holzbauer, S, Dubray, C, Guillaumot, L: Zika virus outbreak on yap island, Federated States of Micronesia. N. Engl. J. Med. 2009(360), 2536-2543 (2009)

21. Bearcroft, W: Zika virus infection experimentally induced in a human volunteer. Trans. R. Soc. Trop. Med. Hyg. 50(5), 442-448 (1956)

22. Fleming, WH, Rishel, RW: Deterministic and Stochastic Optimal Control, vol. 1. Springer, New York (2012)

23. Lenhart, S, Workman, JT: Optimal Control Applied to Biological Models. CRC Press, Boca Raton (2007)

24. Mosquito control: can it stop Zika at source? World Health Organization (WHO).

http://www.who.int/emergencies/zika-virus/articles/mosquito-control/en/ (2016)

\section{Submit your manuscript to a SpringerOpen ${ }^{\circ}$} journal and benefit from:

- Convenient online submission

- Rigorous peer review

- Open access: articles freely available online

- High visibility within the field

- Retaining the copyright to your article

Submit your next manuscript at $\boldsymbol{~ s p r i n g e r o p e n . c o m ~}$ 